\title{
Current treatment options for newly diagnosed metastatic hormone-sensitive prostate cancer-a narrative review
}

\author{
Sean Ong ${ }^{1,2} \wedge$, Jonathan O'Brien ${ }^{1,3}$, Elizabeth Medhurst $^{1}$, Nathan Lawrentschuk ${ }^{1,2,3}$, \\ Declan Murphy ${ }^{1,4}$, Arun Azad ${ }^{4,5}$
}

${ }^{1}$ Division of Cancer Surgery, Peter MacCallum Cancer Centre, Melbourne, Victoria, Australia; ${ }^{2}$ EJ Whitten Foundation Prostate Cancer Research Centre, Epworth Health, Victoria, Australia; ${ }^{3}$ Department of Urology, Royal Melbourne Hospital, Parkville, Victoria, Australia; ${ }^{4}$ Sir Peter MacCallum Department of Oncology, University of Melbourne, Parkville, Victoria, Australia; ${ }^{5}$ Department of Medical Oncology, Peter MacCallum Cancer Centre, Melbourne, Victoria, Australia

Contributions: (I) Conception and design: D Murphy, A Azad; (II) Administrative support: None; (III) Provision of study materials or patients: None; (IV) Collection and assembly of data: All authors; (V) Data analysis and interpretation: None; (VI) Manuscript writing: All authors; (VII) Final approval of manuscript: All authors.

Correspondence to: Associate Professor Arun Azad. Peter MacCallum Cancer Centre, 305 Grattan Street, Parkville, 3000, Victoria, Australia.

Email: arun.azad@petermac.org.

\begin{abstract}
Prostate cancer continues to be one of the most commonly diagnosed cancers in men globally and a leading cause of male cancer deaths. The landscape of metastatic hormone-sensitive prostate cancer has significantly changed over the past decade. For many years, androgen deprivation therapy alone through surgical or chemical castration was the mainstay of treatment yielding limited 5-year survival rates. New treatment approaches using Docetaxel chemotherapy or androgen receptor pathway inhibitors to intensify upfront systemic therapy have resulted in significantly improved survival rates compared to androgen deprivation therapy alone. Clinicians are now equipped with an arsenal of drugs capable of prolonging life for metastatic hormone-sensitive prostate cancer patients. Furthermore, new treatment modalities are being tested in clinical trials making treatment of metastatic hormone-sensitive prostate cancer an extremely dynamic space. In this narrative review, we provide an overview of the key systemic treatments for metastatic hormone-sensitive prostate cancer, namely androgen deprivation therapy, novel androgen receptor pathway inhibitors and Docetaxel. We summarise a series of landmark trials that have led to the integration of novel androgen receptor pathway inhibitors and docetaxel into the treatment paradigm for metastatic hormonesensitive prostate cancer. Lastly, we discuss nursing, financial and side-effect considerations pertaining to the use of these drugs. This article aims to give its readers an understanding of the evidence and clinical aspects of novel therapies in metastatic hormone-sensitive prostate cancer as they become increasingly available for use around the world.
\end{abstract}

Keywords: Prostate cancer; metastatic; hormone-sensitive; management

Submitted Jul 29, 2020. Accepted for publication Jan 05, 2021.

doi: $10.21037 /$ tau-20-1118

View this article at: http://dx.doi.org/10.21037/tau-20-1118

$\wedge$ ORCID: 0000-0003-1117-2409. 


\section{Introduction}

Prostate cancer incidence has risen all around the world, including in the Asia-Pacific region where it now accounts for more than 120,000 deaths per annum (1). However, incidence varies with low rates observed in South and East Asia, contrasted with higher rates observed in South East Asia, and among the highest rates in the world reported in Australia (2). Despite being extremely treatable in its early stages, prostate cancer still remains a leading cause of cancer-related death worldwide (3), and in Australia, is the second leading cause of male cancer-related deaths (4). Of these men, approximately one third presented with newly diagnosed or de novo metastatic prostate cancer and two thirds relapsed after prior local therapy (5). Advanced stage prostate cancer continues to have a poor prognosis which is reflected by a five year survival rate of $31-36 \%(6,7)$.

Androgen deprivation therapy (ADT) has been at the forefront of treatment for metastatic prostate cancer since the Nobel prize winning works of Huggins and Hodges. Unfortunately, although effective at first, all patients inevitably develop a rising PSA or new metastases despite castrate levels of testosterone. This marks the transition to a lethal form of prostate cancer known as castrationresistant prostate cancer (CRPC). The discovery of new androgen receptor pathway inhibitors (ARPIs) and the utility of taxane based chemotherapy in the CRPC setting has resulted in significant gains in overall survival (OS). These agents have more recently been tested for metastatic hormone-sensitive prostate cancer (mHSPC), with all of Docetaxel, Abiraterone, Enzalutamide and Apalutamide yielding significant improvements in OS. This has led to changes in treatment guidelines, approvals and subsidies across the globe.

Given the number of options at our disposal, it is paramount that clinicians have an understanding of new agents, their side effect profile and when to prescribe them. Here we review the current treatment options for mHPSC, discuss decision-making considerations and outline future clinical trials that may alter management of this condition.

We identified the treatment options available for use in mHSPC treatment as well as important clinical aspects including nursing, financial and other decision-making considerations. A search was then performed in PubMed and Google Scholar for the most recent and relevant articles pertaining to the topics identified. An emphasis was placed on phase 3 clinical trials for inclusion into our article. Clinicaltrials.gov was utilised to identify ongoing and future trials for mHSPC. We present the following article in accordance with the Narrative Review reporting checklist (available at http://dx.doi.org/10.21037/tau-20-1118).

\section{Traditional androgen deprivation therapy agents (Table 1)}

\section{LHRH agonist}

Luteinizing hormone-releasing hormone (LHRH, also known as gonadotropin releasing hormones) is released by the hypothalamus and stimulates the anterior pituitary gland to release luteinizing hormone (LH) and folliclestimulating hormone (8) ultimately producing testosterone. It was first discovered and then isolated by two Nobel prize winners-Schally and Guillemin - in the late 1960s/ early 1970s. Over the next 20 years LHRH was recognised as being a clinically relevant target for hormone-sensitive cancers such as breast cancer and prostate cancer. Pioneers in the field made significant advancements that culminated in the approval of the first LHRH agonist (triptorelin) for use in prostate cancer in 1986 (9).

LHRH agonists disrupt the normally pulsatile release of LHRH from the hypothalamus and over time result in a downregulation of $\mathrm{LH}$ with testosterone reaching castration levels at around 3-4 weeks (10). Unfortunately, there is an initial flare or surge of testosterone causing a transient increase in tumour volume. This can have a clinical effect causing urinary obstruction, bone pain and even spinal cord compression (11). The effects of testosterone flare can be mitigated by the short-term use of a first-generation antiandrogen such as bicalutamide.

\section{LHRH antagonists}

The development of LHRH antagonists combatted the initial "flare" or "surge" effect of testosterone from LHRH agonists. LHRH antagonists bind directly to LHRH receptors in the pituitary, thus provoking an immediate blockade of $\mathrm{LH}$ and a fast decrease in testosterone levels without the flare phenomenon (11). Abarelix was the first approved LHRH antagonist, however studies found significant clinical adverse effects such as severe hypersensitivity reactions and escape from castration that subsequently led to its cessation of use $(7,12)$. Since then, a new and improved third generation LHRH antagonist, degarelix, has become the mainstay of LHRH antagonist treatment. Klotz et al. compared the efficacy and tolerability 
Table 1 Medications for mHSPC

\begin{tabular}{|c|c|c|c|}
\hline Class of drug & Common & Mechanism of action & Adverse effects \\
\hline Steroidal ARPIs & Abiraterone & $\begin{array}{l}\text { Inhibits P450 c17 (CYP17)-a critical } \\
\text { enzyme in androgen synthesis pathway }\end{array}$ & Cardiac and hepatic toxicity, hypertension \\
\hline $\begin{array}{l}\text { Taxane-based } \\
\text { chemotherapy }\end{array}$ & $\begin{array}{l}\text { Docetaxel, } \\
\text { Cabazitaxel }\end{array}$ & $\begin{array}{l}\text { Binds to protein (tubulin) found in } \\
\text { microtubules of cells }\end{array}$ & $\begin{array}{l}\text { Neutropenia, alopecia, nail changes, sensory } \\
\text { neuropathy, peripheral oedema, diarrhoea }\end{array}$ \\
\hline
\end{tabular}

LHRH, luteinizing hormone releasing hormone; ARPI, androgen receptor pathway inhibitors; mHSPC, metastatic hormone-sensitive prostate cancer.

of degarelix to leuprolide in 610 men with hormonesensitive prostate cancer. The authors found that $>95 \%$ patients taking degarelix were able to reach castration levels (testosterone $<0.5 \mathrm{ng} / \mathrm{mL}$ ) by day 3 . Degarelix also displayed the same ability to maintain these levels over 1 year compared to LHRH agonists (13). Most recently, an oral third generation LHRH antagonist relugolix was proven to be superior to leuprolide in achieving rapid and sustained testosterone suppression (14). Over $96 \%$ of men who received relugolix maintained castration through to 48 weeks as compared to $88 \%$ in the leuprolide group. Moreover, $56 \%$ of men reached castration levels at day 4 with relugolix (14). Of particular note, relugolix was also associated with a $54 \%$ relative risk reduction in major adverse cardiovascular events as compared to leuprolide.

\section{Adverse effects}

In spite of their proven benefit for prostate cancer patients, traditional ADT agents can have considerable adverse effects pertaining to their hormonal manipulation. ADT decreases serum testosterone levels by $>95 \%$ and by doing so gives rise to a number of physiological alterations. A change in bone and muscle homeostasis, lipid profile, hypertension and insulin sensitivity can lead to fractures, diabetes and cardiovascular events $(14,15)$. Other side effects include hot flushes, gynaecomastia, sexual dysfunction and mental health issues (16).

\section{Androgen receptor pathway inbibitors (ARPI)}

The initial management for metastatic prostate cancer is surgical or medical castration via ADT. Nearly all patients initially respond but resistance is inevitable, indicated by a rising PSA level, new lesions, and/or progressive symptoms. This lethal phenotype is referred to as CRPC but remains driven by persistent androgen receptor (AR) signalling (17). Numerous mechanisms have been implicated in persistent AR signalling in CRPC including AR gene amplification, AR gene mutations, AR splice variants, extra-gonadal androgen synthesis, increased expression of AR coregulators and non-AR signalling pathways that cross-talk with the AR. A greater understanding of the role of the $\mathrm{AR}$ in development of CRPC underpinned efforts to more effectively target androgen signalling using ARPIs.

ARPIs have been investigated and refined since the 1960s. Steroidal ARPIs have a similar chemical structure to testosterone and progesterone and therefore can have unintended hormonal side effects not relating to prostate cancer treatment. Non-steroidal ARPIs have a 
distinct chemical structure and therefore do not exhibit progesterone related side effects and are more tailored as a pure AR blocker.

Non-steroidal ARPIs have progressed through decades of research. They exert their effect at a cellular level by competitively binding to the androgen receptor thereby inhibiting testosterone and dihydrotestosterone from activating their biological reactions. First generation nonsteroidal ARPIs such as bicalutamide, although effective at first, had flaws. They have a low affinity for the AR (18) but perhaps most concerning was their agonist effect that were in some cases when castration resistance ensued $(19,20)$.

Second generation non-steroidal ARPI-such as Enzalutamide, Apalutamide and Darolutamide-have a greater affinity for the AR than first-generation ARPIs and have no known or minimal agonistic effects. Along with being a competitive inhibitor of the $\mathrm{AR}$, these agents combat increased AR gene expression by inhibiting nuclear translocation of the AR, DNA binding, and coactivator recruitment (21). Enzalutamide showed superior OS in both chemotherapy-treated and chemotherapy-naïve mCRPC patients $(21,22)$. Apalutamide is a non-steroidal ARPI with the same mechanism of action as Enzalutamide but the added benefit of having less penetration of the blood brain barrier. In addition to this, early evidence suggests that apalutamide may not increase the frequency of AR anomalies that contribute to castrate resistance (23). Darolutamide is another second generation non steroidal ARPI that is a potent AR inhibitor but is structurally different from Enzalutamide and Apalutamide. This accounts for its negligible blood brain barrier penetration and theoretically less CYP drug interactions in pre-clinical models (24).

Abiraterone is a second generation steroidal ARPI that inhibits a critical enzyme in androgen synthesis named P450 c17 (CYP17). By doing so, Abiraterone is able to decrease testosterone produced by the adrenal gland and also intracellularly in prostate cancer cells. It has also shown antagonistic and downregulatory effects of AR (25). Like Enzalutamide, Abiraterone has demonstrated significant improvements in OS for both chemotherapy-treated and chemotherapy-naïve mCRPC patients $(26,27)$.

\section{Taxane based chemotherapy}

Taxane based chemotherapy has been the mainstay of chemotherapy for metastatic prostate cancer since 2004 when Docetaxel was shown to significantly improve OS in mCRPC over mitoxantrone in the TAX-327 trial (28). Taxanes act by binding to tubulin, a protein found in microtubules of cells. By doing so, taxanes stabilise the microtubules not allowing them to disassemble and carry out their normal functions including vesicle transport, transcription factor trafficking, cell signalling and chromosome separation during cell division (mitosis) (29).

\section{Combination trials (Table 2)}

Given the proven activity of Docetaxel and ARPIs in mCRPC, multiple clinical trials were then initiated to look at the activity of these agents when used upfront with ADT in mHSPC. Although key differences exist between these trials in regards to treatment combinations and inclusion of specific patient sub-groups, overall these studies have shown a major benefit with intensification of upfront systemic treatment of mHSPC. Of note, the definition of high volume/high risk metastatic prostate cancer has not yet been universally defined. The two main criteria derived from the CHAARTED trial and LATITUDE trial is shown in Table 3.

\section{Docetaxel plus ADT}

Three large randomised trials have studied the efficacy of Docetaxel compared to standard therapy in mHSPC patients.

The GETUG-AFU 15 trial was a randomised phase 3 trial run in 29 centres throughout France and Belgium. 385 men with mHPSC were recruited and were randomised to receive ADT plus a non-steroidal ARPI or Docetaxel $\left(75 \mathrm{mg} / \mathrm{m}^{2}\right.$ intravenously on the first day of each 21-day cycle; up to nine cycles) without prednisone. In this study, with a median follow up of 50 months, there was no statistically significant difference in OS between the two treatment arms. However, biochemical and radiographic PFS was significantly prolonged in the Docetaxel arm (30). An updated report of the analysis revealed that at median follow up of 83.9 months, median OS was 62.1 months and 48.6 months in the Docetaxel plus ADT and ADT arms respectively (31). These conflicting results may have been due to the inclusion of a relatively large percentage of low volume metastatic disease (52\% low volume disease defined using CHAARTED criteria). In the Docetaxel plus ADT group, 39 of 189 (21\%) patients discontinued treatment due to toxicity effects. Neutropenia (32\% of patients in this arm), febrile neutropenia (7\%), fatigue (7\%), erectile 


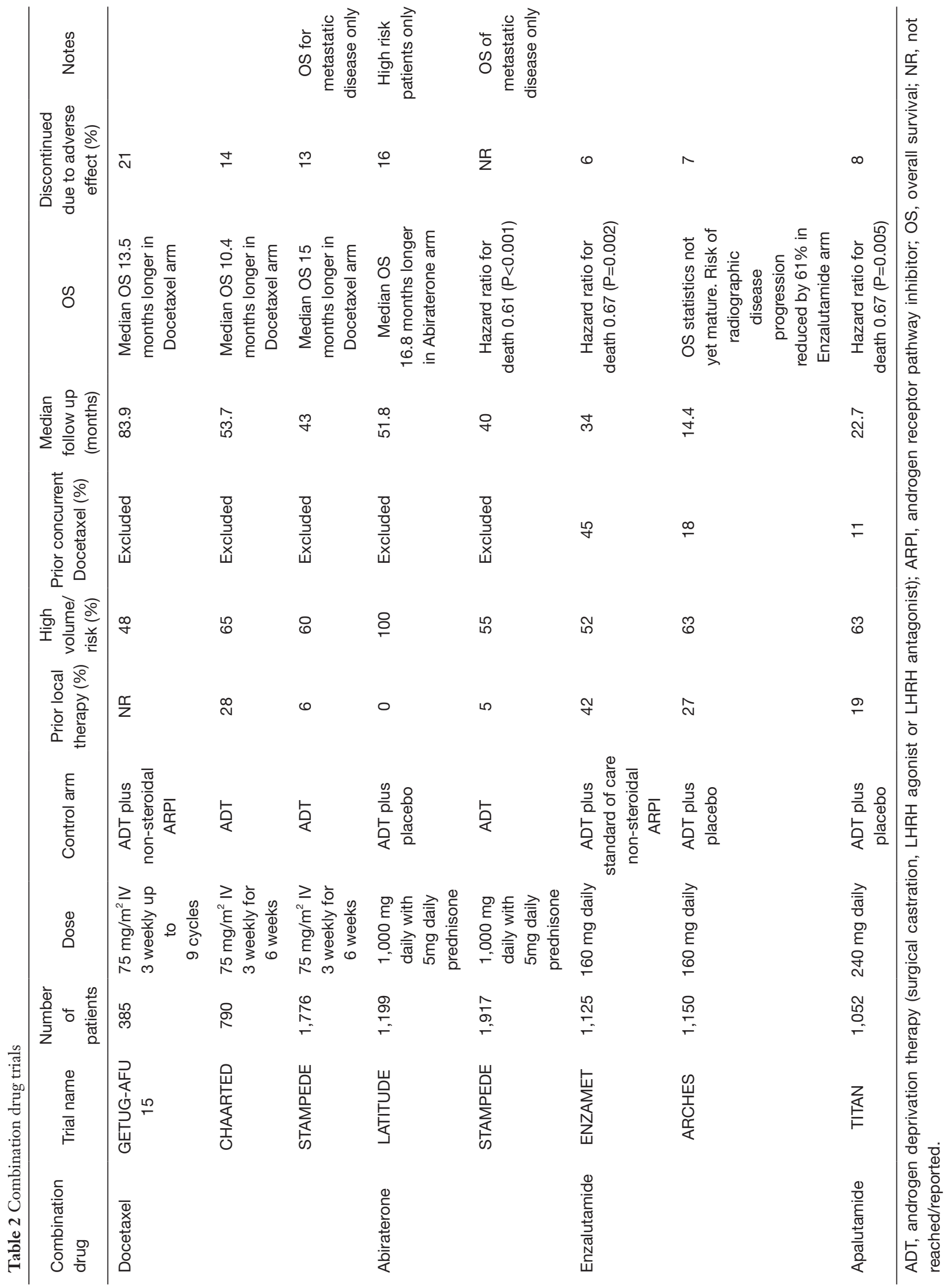


Table 3 High volume/risk criteria for metastatic prostate cancer

\begin{tabular}{ll}
\hline CHAARTED high volume criteria & LATITUDE high risk criteria \\
\hline$>4$ bone metastases (at least one outside the spine or pelvis & Two or more of the following criteria: \\
AND/OR Visceral metastases & $\bullet>3$ bone metastases \\
& $\bullet$ Gleason score $>8$ \\
& $\bullet$ Visceral metastases (not including lymph node metastases) \\
\hline
\end{tabular}

dysfunction (8\%), and decreased libido (6\%) were the most common grade 3-4 adverse effects recorded (32).

The CHAARTED trial enrolled 790 men with mHSPC to receive either ADT or ADT plus Docetaxel $\left(75 \mathrm{mg} / \mathrm{m}^{2}\right.$ intravenously every 3 weeks for six weeks) plus dexamethasone. In both arms, median age of patients were around 63-64 years and approximately $70 \%$ of patients had ECOG performance status of 0 . Around $65 \%$ of patients had high volume disease and in both arms, $72.8 \%$ of patients had received no previous local therapy and therefore were considered to have de novo metastatic disease. At median follow up of 28.9 months, median OS was found to be 13.6 months longer in the Docetaxel arm (56.7 vs. 44 months). The median time to clinical progression was found to be 33 months in the Docetaxel arm compared to 19.8 months in the ADT only arm (32). More mature results at median follow up of 53.7 months showed median OS was 10.4 months longer in the Docetaxel arm for all patients. Survival benefit was more pronounced in high volume disease patients (16.8 months longer OS in Docetaxel arm), however in contrast survival benefit in low volume disease was not demonstrated (33). It should be noted that the statistical design of CHAARTED was not powered to show a survival benefit in patients with low volume disease. In this trial, $86 \%$ of patients completed six cycles of Docetaxel therapy. Neutropenia (12\%), febrile neutropenia (6\%), fatigue (4\%), infection with neutropenia (2\%) and allergic reaction (2\%) were the most common grade 3-4 adverse effects reported in the Docetaxel arm.

The STAMPEDE trial is the largest and most recent trial to analyse the effects of Docetaxel. In a multi-arm, multistage design, the STAMPEDE group recruited 2,962 men with hormone-sensitive prostate cancer to be randomised to receive $\mathrm{ADT}$, $\mathrm{ADT}$ plus Docetaxel $\left(75 \mathrm{mg} / \mathrm{m}^{2}\right.$ intravenously every 3 weeks for six weeks) plus prednisolone, ADT plus zoledronic acid or ADT plus Docetaxel plus zoledronic acid. Median age of men in all arms were around 65 years and almost all men (94\%) had newly diagnosed prostate cancer.
$61 \%$ of men had metastatic disease and the remaining $39 \%$ had locally advanced disease. Zoledronic acid was not shown to have any survival benefit. At median follow up of 43 months, the Docetaxel arm and Docetaxel plus zoledronic acid arm showed 10 and 5 months respectively longer median OS compared to ADT for all patients. In a subgroup analysis of patients with metastatic disease, the Docetaxel arm showed median OS was 15 months longer compared to ADT only (34). The Docetaxel and Docetaxel plus zoledronic acid arms both recorded $13 \%$ of patients discontinue treatment due to toxicity. Adverse effects were also similar in both arms with febrile neutropenia (15\%), endocrine disorders (10-12\%), neutropenia (10\%), general disorders (7-11\%), and gastro-intestinal disorders (7-8\%) being the most commonly reported grade 3-4 adverse effects (34).

\section{Abiraterone plus ADT}

Abiraterone was the first of the novel ARPIs to be trialled and proven in the mHSPC setting.

The LATITUDE trial was an international placebo controlled, randomised phase 3 trial analysing Abiraterone. This group assigned 1,199 men with high risk mHSPC (at least two of the following prognostic factors: Gleason score 8 or higher, 3 or more bone metastases, or measurable visceral metastases) to receive ADT plus Abiraterone 1,000 mg daily plus prednisone $5 \mathrm{mg}$ daily versus placebo. At a median follow up of 30.4 months, the 3 -year OS rate was $66 \%$ and $49 \%$ in the Abiraterone arm and placebo arm respectively [hazard ratio (HR) for death $=0.62,95 \%$ confidence interval (CI): $0.51-0.76, \mathrm{P}<0.001]$. Median PFS was 33 vs. 14.8 months in the Abiraterone arm and placebo arm respectively (HR 0.47, 95\% CI: 0.39-0.55, $\mathrm{P}<0.001$ (27). A final analysis at median follow up of 51.8 months confirmed Abiraterone's superiority over ADT alone. Median OS was significantly longer in the Abiraterone vs. placebo arm (53.3 vs. 36.5 months; HR 0.66, 
95\% CI: $0.56-0.78, \mathrm{P}<0.0001)(35)$. In the Abiraterone group, $16 \%$ of patients discontinued treatment due to adverse effects as compared to $10 \%$ in the placebo group. The most common grade 3-4 adverse effects in the Abiraterone group were hypertension (21\% of patients), hypokalaemia (12\%) and liver transaminase increase (5\%).

The STAMPEDE trial group also incorporated an arm comparing Abiraterone plus prednisone to ADT. 1,917 men with hormone-sensitive prostate cancer were randomised to receive ADT with or without Abiraterone $1,000 \mathrm{mg}$ daily plus prednisone $5 \mathrm{mg}$ daily. In both arms, median age of men was 67 years and $78 \%$ of men had WHO performance status of 0 . Metastatic disease patients occupied approximately $50 \%$ of both arms. Of these patients, $88 \%$ of men had bone metastases and over $95 \%$ were newly diagnosed cancer. At median follow up of 40 months, the Abiraterone group showed an improved 3 -year survival rate of $83 \%$ compared to $76 \%$ in the $\mathrm{ADT}$ only group (HR for death $=0.63$, 95\% CI: 0.52-0.76, $\mathrm{P}<0.01)$. In the subgroup analysis of mHSPC patients only, Abiraterone again demonstrated improved 3 year OS (HR 0.61, 95\% CI: $0.49-0.75, \mathrm{P}<0.001)$ and improved 3 year PFS (HR 0.40, 95\% CI: 0.34 to 0.47, P<0.001) (36). Grade 3-4 adverse effects were reported in $47 \%$ of the Abiraterone group compared to $33 \%$ in the ADT only group. The most common adverse effects in the Abiraterone group were endocrine disorders (14\%), musculoskeletal disorders (7\%), increased hepatic transaminases (7\%), hypertension (5\%) and gastro-intestinal disorders (5\%).

In regards to adverse effects, pooled analysis of results from the STAMPEDE trial and LATITUDE trial showed that Abiraterone exhibited an approximately three fold increase in grade 3-4 acute cardiac and hepatic toxicity and a two fold increase in grade 3-4 vascular events relating to hypertension (37). Abiraterone therefore should be prescribed with caution or close follow up in patients with pre-existing hypertension, cardiac disease and liver disease. Clinicians should also be mindful of diabetic patients as Abiraterone needs to be taken in combination with prednisone.

\section{Enzalutamide plus ADT}

ENZAMET was an international, open label, randomised, phase 3 trial of 1,125 men with mHSPC. These men were randomised in 1:1 ratio to either receive Enzalutamide $160 \mathrm{mg}$ daily or a standard care nonsteroidal drug (bicalutamide, nilalutamide or flutamide) with background continuous testosterone suppression. Median age of men in both arms was 69 years. $52 \%$ of men in the Enzalutamide arm had high volume disease as compared to $53 \%$ in the standard of care arm. Docetaxel was given to $17 \%$ and $15 \%$ of the Enzalutamide group and standard care group respectively. At a median follow up of 34 months, there were 102 deaths $v s .134$ deaths in the Enzalutamide arm and standard of care arm respectively (HR for death 0.67, 95\% CI: 0.52-0.86, $\mathrm{P}=0.002)$. The trial was not yet able to state median survival time. 3-year OS rates were estimated to be $80 \%$ and $72 \%$ in Enzalutamide group vs. standard care group respectively. PSA PFS rates were $67 \%$ vs. $37 \%$ in the Enzalutamide group vs. standard care group respectively (HR 0.39, $95 \%$ CI: $0.33-0.47, \mathrm{P}<0.001)$. Clinical PFS rates were $68 \%$ and $41 \%$ in Enzalutamide group vs. standard care group respectively (HR 0.40, 95\% CI: 0.33-0.49, P<0.001). Interestingly, subgroup analysis of men with high volume disease (HR 0.74, 95\% CI: 0.55-1.01, $\mathrm{P}=0.04$ ), and planned early Docetaxel treatment (HR 0.91, 95\% CI: 0.62-1.35, $\mathrm{P}=0.04$ ) found that Enzalutamide's effect on OS was less pronounced (38). Approximately $6 \%$ of patients in the Enzalutamide arm discontinued treatment due to adverse effects compared to $3 \%$ in the standard of care arm. Febrile neutropenia (7\%), hypertension (8\%), fatigue $(6 \%)$, and syncope (4\%) were the most common grade 3-5 adverse effects recorded. Of note, all but two events of febrile neutropenia occurred during early Docetaxel treatment and also only 2 events of seizures was recorded (38).

ARCHES trial was an international, randomised, phase 3 trial that randomised 1,150 men with $\mathrm{mHSPC}$ in a $1: 1$ ratio to either Enzalutamide or placebo with ADT. Overall, the median age of these men was 70 years and over $75 \%$ of the men had ECOG performance status of $0.63 .2 \%$ of these men had high volume disease and $17.9 \%$ had received prior Docetaxel chemotherapy. These characteristics were well balanced between the two study groups. Preliminary results at median follow up of 14.4 months have shown Enzalutamide reduced risk of radiographic disease progression or death by $61 \%$ compared to the placebo arm (HR 0.39, 95\% CI: 0.30-0.50, $\mathrm{P}<0.001)$. Median radiographic PFS was not reached with Enzalutamide $v s$. 19 months for the placebo group. Subgroup analysis of high and low volume disease and patients with prior Docetaxel treatment showed similar results across all groups. OS statistics are not yet mature (39). Discontinuation of Enzalutamide due to adverse effects occurred in $7 \%$ of patients compared to $5 \%$ in the placebo group. Grade 3 or above adverse effects recorded included hypertension (3\%), fatigue (1.6\%), 
musculoskeletal events (1.6\%) and convulsions (0.3\%).

\section{Apalutamide plus ADT}

The TITAN trial remains the only study analysing Apalutamide in the mHSPC setting. 1,052 men were recruited then assigned to either receive ADT plus Apalutamide or placebo. This cohort of men had a median age of 68 years; $11 \%$ of men had received previous Docetaxel and $16.4 \%$ of patients previous prostatectomy or radiotherapy for localised disease. $63 \%$ of men had high volume disease and the remainder had low volume disease. At median follow up of 22.7 months, the OS percentage was greater in the Apalutamide arm ( $82 \%$ vs. $74 \%$; HR for death 0.67 , 95\% CI: $0.51-0.89, \mathrm{P}=0.005)$ and there was no significant difference in this result when analysing according to disease volume. Apalutamide also performed better when assessing for radiographic PFS (68\% vs. 48\%; HR for radiographic progression or death $0.48,95 \%$ CI: $0.39-0.60, \mathrm{P}<0.001)(40)$. Adverse effects led to discontinuation of Apalutamide in $8 \%$ of patients compared to $5 \%$ in the placebo group. Grade 3 or above fatigue $(1.9 \%)$ and rash $(2.7 \%)$ were noticeably higher in the Apalutamide group compared to the placebo group. Grade 1-2 hypothyroidism (6.5\%) was also noticeably increased in the Apalutamide arm (41).

\section{Treatment choice}

Four agents have now demonstrated OS and PFS benefits in patients with mHSPC. A recent meta-analysis of the above trials attempted to indirectly compare the efficacy of each combination approach however found no statistically significant differences in OS (41). Therefore, these practice changing results have now given clinicians several treatment options for mHSPC.

For now, treatment selection should be tailored to each individual case with differing adverse effect profiles of each medication at the forefront of management considerations. These are based on the reported rates of adverse effects from the trials above. In essence, patients with poorly controlled heart disease, diabetes, hypertension or liver disease should not be offered Abiraterone. Patients who have had a major stroke, moderate-severe cognitive issues or seizures should not be offered enzalutmide. And when considering taxane based chemotherapy, contraindications include poor performance status neuropathy, dementia, uncontrolled pre-existing serious disease, severe hepatic or renal insufficiency and neutropenia (42). Thought should also be given to patient lifestyle and housing location when deciding between frequent hospital visits for intravenous chemotherapy or daily oral ARPIs.

Another key consideration centres around the impact of choice of initial therapy for mHSPC on subsequent treatment selection in mCRPC. A logical approach would be to use docetaxel following prior ARPI therapy, and vice versa. Whether any mHSPC patients would benefit from switching to a second-line ARPI at time of progression to mCRPC is unknown, although currently available data suggests this would be of limited value $(43,44)$. With additional systemic agents likely to enter the treatment paradigm for $\mathrm{mCRPC}$ and $\mathrm{mHSPC}$ in coming years, carefully designed prospective clinical trials and highquality real-world databases will be needed to help address questions around treatment sequencing.

Similarly, the role of combination chemotherapy and ARPIs requires further investigation. Currently, there is no data to support concurrent treatment with docetaxel and an ARPI. In fact, in ENZAMET triplet therapy (ADT + Docetaxel + Enzalutamide) appeared to be associated with higher rates of adverse events including neuropathy (38). It must be said however that ENZAMET was not designed to formally assess the combination of Docetaxel and Enzalutamide. Two ongoing trials are assessing the combination of Docetaxel and an ARPI, namely ARASENS (Docetaxel +/- DarolutamideNCT02799602) (45) and PEACE-1 (Docetaxel +/Abiraterone-NCT01957436). The results of these trials are eagerly awaited and will help to define the benefit, if any, of concurrent treatment with Docetaxel and an ARPI in mHSPC.

Additionally, there have been some indications that volume of disease, age and cost effectiveness could have a role to play in stratifying patients for each drug. However, definitions for volume and age need to be standardised and further investigated and costs will most likely change in the coming years. Furthermore, the impact of novel imaging using prostate specific membrane antigen (PSMA) is not yet established. Although PSMA PET/CT is superior to $\mathrm{CT}$ and bone scan for the evaluation of metastases in newly diagnosed patients (46) which may lead to stage migration of mHSPC patients, it is not yet clear if this will lead to improved oncological outcomes (47). Certainly, nuclear medicine physicians now play a pivotal role in the prostate cancer multidisciplinary team (48).

\section{The role of the urologist}

Management of mHSPC has traditionally been in the 
domain of the urologist. Since ADT was standardised as a first-line option for these patients many years ago, it has been the urologist who has been the primary specialist overseeing their care until the emergence of castrationresistance. However, the upfront use of Docetaxel chemotherapy and ARPIs has led to changes in patterns of care, with medical oncologists playing an increasing role in the primary management of mHSPC. Whilst Docetaxel is most often administered by medical oncologists, it is also administered by urologists in some countries, especially in the Asia-Pacific region (2). The increasing role for well tolerated oral ARPIs combined with ADT for mHSPC, also means that urologists can continue to play a key role in the management of mHSPC, provided they are willing to become facile with the safe use and monitoring of these agents (49). Ultimately, these patients are best managed in multidisciplinary teams, taking into account patient and disease factors, as well as access and affordability issues (50).

\section{Nursing considerations}

With a number of treatments now available for men with metastatic prostate cancer, the role of a nurse is vital in the ongoing coordination and management of patient care and the improvement of quality of life (51). Nurses have the ability to develop relationships with the patient and their families whilst also creating support networks tailored to the individual's lifestyle and preferences (52). Information relating to age, comorbidities, past experiences, reliability and financial status can then be available for clinicians to consider when decision-making regarding appropriate treatment $(51,53)$. This information also leads to early identification of referrals to allied health, culminating in improved patient tolerability of treatment and ultimately resulting in greater quality of life and overall experience (53). Although studies have shown patients benefit from the involvement of nursing coordination, health organisations still inconsistently utilise this resource. Further research and standardisation of nursing care is required to maximise its role in prostate cancer survivorship (51).

\section{Cost}

The financial burden of advanced prostate cancer can have a significant impact on cancer survivorship (54). Although Abiraterone and Enzalutamide have now demonstrated an improved survival benefit, Docetaxel remains the more cost effective option. This was confirmed in a statistical analysis by Sathianathen et al. who showed in a cohort of men from the US, ADT plus Abiraterone demonstrated clinically better results compared to ADT plus Docetaxel but conversely ADT plus Docetaxel was the more cost-effective treatment option (55). Moving forward, reimbursement status and affordability of these drugs will change as evidence for them grows larger. Nevertheless, a holistic approach should be implemented when discussing the correct treatment plan for each patient.

\section{Other approaches}

\section{Treatment of the primary tumour}

The last decade has seen much debate amongst prostate cancer specialists in regard to cytoreductive treatment for the primary tumour. Two large trials have revealed answers pertaining to external beam radiotherapy (EBRT) in this space, however, the specific cohorts of patients that would benefit from ERBT and the field of ERBT used should be further refined. Furthermore, studies comparing cytoreductive prostatectomy to standard treatment need to be carried out.

The evidence for ERBT to the primary tumour is primarily derived from the STAMPEDE trial and HORRAD trial with results from the PEACE-1 trial (56) eagerly awaited. The HORRAD trial recruited 432 men with PSA $>20 \mathrm{ng} / \mathrm{mL}$ and primary bone metastases on whole body bone scan. These men were randomised to receive ADT with or without ERBT (only prostate and any extra-prostatic tumour extension were targeted, pelvic lymph nodes were not). With median follow up of 47 months, improvement in OS was not shown with added ERBT. However in a subgroup analysis of patients with low metastatic burden ( $<5$ metastases), radiotherapy resulted in a non-significant improvement in OS (HR 0.68, 95\% CI: 0.42-1.10) (57). The STAMPEDE trial randomised 2061 men with newly diagnosed mHSPC to receive standard therapy (ADT +/Docetaxel) with or without ERBT (prostate only). Around $60 \%$ of these men had high metastatic burden. Results showed that for all patients, failure free survival was improved but not OS. However, failure free survival (HR 0.59, 95\% CI: 0.49-0.72, $\mathrm{P}<0.0001)$ and OS (HR 0.68, 95\% CI: 0.52-0.90, $\mathrm{P}=0.007)$ were both improved specifically for patients with low metastatic burden $(<4$ bone metastases and no visceral metastases) (58).

In regards to the analysis of the low metastatic burden cohorts in both trials, the similar effect size but lack of 
statistical significance in the HORRAD trial indicates the HORRAD trial was underpowered to show significant difference in this subgroup analysis. At a recent Advanced Prostate Cancer Consensus Conference (APCCC), 98\% of panellists agreed that based on the current literature, local treatment of the primary tumour has an OS benefit only in patients with newly diagnosed, low-volume/low-burden mHSPC (50).

\section{Theranostics}

Within the field of theranostics, radioligand therapy has seen much development in the last 5 years. This technology builds on the discovery of the pivotal prostate specific membrane antigen (PSMA) found on prostate cancer cells. Radioligand therapy utilises PSMA as a vehicle for the radiometal ${ }^{177}$ Lutetium to penetrate cancer cells and emit energy leading to cell death (59). So far, ${ }^{177}$ Lutetium PSMA-617 has only seen viable results in the mCRPC setting $(60,61)$. The transition of ${ }^{177}$ Lutetium PSMA617 to mHSPC patients is the aim of the UpFrontPSMA trial in Australia (62). The trial is currently in the recruitment phase with the aim of including 140 patients in a randomised phase 2 trial of Docetaxel with or without ${ }^{177}$ Lutetium PSMA-617 in hormone naïve prostate cancer. Their primary objective will be PSA response but will have secondary endpoints analysing PFS (PSA and radiological), time to castration resistance, quality of life and OS.

\section{Conclusions}

Prostate cancer specialists are now armed with several agents that can be utilised for newly diagnosed mHSPC. Docetaxel, Abiraterone, Enzalutamide and Apalutamide have all been proven in large randomised trials to prolong OS compared to previous standard of care ADT. As these new agents become more readily available and subsidised, clinicians should dictate their choice of treatment based on patient preference, co-morbidities, and medication adverse effect profiles. Furthermore, the role of a clinical nurse is important in this decision and can improve patient survivorship.

Along with these novel medications, other approaches including treatment to the primary tumour, and radioligand therapy are currently being investigated for mHSPC. The combination of these therapies will look to push the envelope for advanced prostate cancer treatment and survival.

\section{Acknowledgments}

Funding: None.

\section{Footnote}

Provenance and Peer Review: This article was commissioned by the Guest Editor (Tilman Todenhöfer) for the series "Management of Advanced Genitourinary Malignancies" published in Translational Andrology and Urology. The article has undergone external peer review.

Reporting Checklist: The authors have completed the Narrative Review reporting checklist. Available at http:// dx.doi.org/10.21037/tau-20-1118

Peer Review File: Available at http://dx.doi.org/10.21037/ tau-20-1118

Conflicts of Interest: All authors have completed the ICMJE uniform disclosure form (available at http://dx.doi. org/10.21037/tau-20-1118). The series "Management of Advanced Genitourinary Malignancies" was commissioned by the editorial office without any funding or sponsorship. Dr. DM reports non-financial support and other from Janssen, non-financial support and other from Astellas, non-financial support and other from Bayer, non-financial support and other from Ferring, non-financial support and other from Ipsen, non-financial support and other from Astra Zeneca, outside the submitted work. Dr. AA reports grants and personal fees from Astellas, grants and personal fees from Merck Serono, grants from Astra Zeneca, personal fees from Bristol Myers Squibb, grants from Aptevo Therapeutics, grants from Glaxo Smith Kline, personal fees from Pfizer, grants from MedImmune, grants from SYNthorx, grants from Bionomics, grants from Sanofi Aventis, personal fees from Novartis, personal fees from Janssen, personal fees from Tolmar, personal fees from Amgen, outside the submitted work. The authors have no other conflicts of interest to declare.

Ethical Statement: The authors are accountable for all aspects of the work in ensuring that questions related to the accuracy or integrity of any part of the work are appropriately investigated and resolved.

Open Access Statement: This is an Open Access article distributed in accordance with the Creative Commons 
Attribution-NonCommercial-NoDerivs 4.0 International License (CC BY-NC-ND 4.0), which permits the noncommercial replication and distribution of the article with the strict proviso that no changes or edits are made and the original work is properly cited (including links to both the formal publication through the relevant DOI and the license). See: https://creativecommons.org/licenses/by-nc-nd/4.0/.

\section{References}

1. Culp MB, Soerjomataram I, Efstathiou JA, et al. Recent Global Patterns in Prostate Cancer Incidence and Mortality Rates. Eur Urol 2020;77:38-52.

2. Chiong E, Murphy DG, Akaza H, et al. Management of patients with advanced prostate cancer in the Asia Pacific region: 'real-world' consideration of results from the Advanced Prostate Cancer Consensus Conference (APCCC) 2017. BJU Int 2019;123:22-34.

3. Ferlay J, Colombet M, Soerjomataram I, et al. Estimating the global cancer incidence and mortality in 2018: GLOBOCAN sources and methods. Int J Cancer 2019;144:1941-53.

4. Australia C. Prostate Cancer in Australia statistics 2020. Available online: https://www.canceraustralia. gov.au/affected-cancer/cancer-types/prostate-cancer/ prostate-cancer-australia-statistics\#: :text=In\%20 2016\%2C\%20there\%20were\%2019\%2C305,will\%20 be $\% 20$ diagnosed $\% 20$ in $\% 20$ Australia.\&text=In\%20 2016\%2C\%20the\%20age\%2Dstandardised,141\%20 cases $\% 20$ per\% 20100\%2C000\%20males

5. Welfare AIoHa. Cancer in Australia 2019 Australia2019. Available online: https://www.aihw.gov.au/reports/cancer/ cancer-in-australia-2019/contents/table-of-contents.

6. Team TACSmaec. Survival Rates for Prostate Cancer 2020 [updated 9/1/2025/5/20]. Available online: https://www. cancer.org/cancer/prostate-cancer/detection-diagnosisstaging/survival-rates.html

7. Indicators NCC. Relative survival by stage at diagnosis (prostate cancer) 2019 [updated 1/4/19. Available online: https://ncci.canceraustralia.gov.au/outcomes/relativesurvival-rate/relative-survival-stage-diagnosis-prostatecancer.

8. Afshar-Oromieh A, Malcher A, Eder M, et al. PET imaging with a [68 Ga] gallium-labelled PSMA ligand for the diagnosis of prostate cancer: biodistribution in humans and first evaluation of tumour lesions. Eur J Nucl Med Mol Imaging 2013;40:486-95.

9. Moreau JP, Delavault P, Blumberg J. Luteinizing hormone-releasing hormone agonists in the treatment of prostate cancer: a review of their discovery, development, and place in therapy. Clin Ther 2006;28:1485-508.

10. Thompson IM. Flare Associated with LHRH-Agonist Therapy. Rev Urol 2001;3 Suppl 3:S10-4.

11. van Poppel H, Nilsson S. Testosterone surge: rationale for gonadotropin-releasing hormone blockers? Urology 2008;71:1001-6.

12. Schröder F, Crawford ED, Axcrona K, et al. Androgen deprivation therapy: past, present and future. BJU Int 2012;109 Suppl 6:1-12.

13. Klotz L, Boccon-Gibod L, Shore ND, et al. The efficacy and safety of degarelix: a 12-month, comparative, randomized, open-label, parallel-group phase III study in patients with prostate cancer. BJU Int 2008;102:1531-8.

14. Shore ND, Saad F, Cookson MS, et al. Oral Relugolix for Androgen-Deprivation Therapy in Advanced Prostate Cancer. N Engl J Med 2020;382:2187-96.

15. Taylor LG, Canfield SE, Du XL. Review of major adverse effects of androgen-deprivation therapy in men with prostate cancer. Cancer 2009;115:2388-99.

16. Walker LM, Tran S, Robinson JW. Luteinizing hormone-releasing hormone agonists: a quick reference for prevalence rates of potential adverse effects. Clin Genitourin Cancer 2013;11:375-84.

17. Ryan CJ, Tindall DJ. Androgen receptor rediscovered: the new biology and targeting the androgen receptor therapeutically. J Clin Oncol 2011;29:3651-8.

18. Kolvenbag GJ, Furr BJ, Blackledge GR. Receptor affinity and potency of non-steroidal antiandrogens: translation of preclinical findings into clinical activity. Prostate Cancer Prostatic Dis 1998;1:307-14.

19. Bohl CE, Gao W, Miller DD, et al. Structural basis for antagonism and resistance of bicalutamide in prostate cancer. Proc Natl Acad Sci U S A2005;102:6201-6.

20. Culig Z, Hoffmann J, Erdel M, et al. Switch from antagonist to agonist of the androgen receptor blocker bicalutamide is associated with prostate tumour progression in a new model system. Br J Cancer 1999;81:242-51.

21. Scher HI, Fizazi K, Saad F, et al. Increased survival with enzalutamide in prostate cancer after chemotherapy. $\mathrm{N}$ Engl J Med 2012;367:1187-97.

22. Beer TM, Armstrong AJ, Rathkopf D, et al. Enzalutamide in Men with Chemotherapy-naïve Metastatic Castrationresistant Prostate Cancer: Extended Analysis of the Phase 3 PREVAIL Study. Eur Urol 2017;71:151-4.

23. Smith MR, Thomas S, Chowdhury S, et al. Androgen 
receptor (AR) anomalies and efficacy of apalutamide (APA) in patients (pts) with nonmetastatic castration-resistant prostate cancer (nmCRPC) from the phase 3 SPARTAN study. Proceedings: AACR Annual Meeting 2018; April 14-18, 2018; Chicago, IL.

24. Moilanen AM, Riikonen R, Oksala R, et al. Discovery of ODM-201, a new-generation androgen receptor inhibitor targeting resistance mechanisms to androgen signalingdirected prostate cancer therapies. Sci Rep 2015;5:12007.

25. Helsen C, Van den Broeck T, Voet A, et al. Androgen receptor antagonists for prostate cancer therapy. Endocr Relat Cancer 2014;21:T105-18.

26. Fizazi K, Scher HI, Molina A, et al. Abiraterone acetate for treatment of metastatic castration-resistant prostate cancer: final overall survival analysis of the COU-AA-301 randomised, double-blind, placebo-controlled phase 3 study. Lancet Oncol 2012;13:983-92.

27. Ryan CJ, Smith MR, Fizazi K, et al. Abiraterone acetate plus prednisone versus placebo plus prednisone in chemotherapy-naive men with metastatic castrationresistant prostate cancer (COU-AA-302): final overall survival analysis of a randomised, double-blind, placebocontrolled phase 3 study. Lancet Oncol 2015;16:152-60.

28. Tannock IF, de Wit R, Berry WR, et al. Docetaxel plus prednisone or mitoxantrone plus prednisone for advanced prostate cancer. N Engl J Med 2004;351:1502-12.

29. Fitzpatrick JM, de Wit R. Taxane mechanisms of action: potential implications for treatment sequencing in metastatic castration-resistant prostate cancer. Eur Urol 2014;65:1198-204.

30. Gravis G, Fizazi K, Joly F, et al. Androgen-deprivation therapy alone or with docetaxel in non-castrate metastatic prostate cancer (GETUG-AFU 15): a randomised, openlabel, phase 3 trial. Lancet Oncol 2013;14:149-58.

31. Gravis G, Boher JM, Joly F, et al. Androgen Deprivation Therapy (ADT) Plus Docetaxel Versus ADT Alone in Metastatic Non castrate Prostate Cancer: Impact of Metastatic Burden and Long-term Survival Analysis of the Randomized Phase 3 GETUG-AFU15 Trial. Eur Urol 2016;70:256-62.

32. Sweeney CJ, Chen YH, Carducci M, et al. Chemohormonal Therapy in Metastatic Hormone-Sensitive Prostate Cancer. N Engl J Med 2015;373:737-46.

33. Kyriakopoulos CE, Chen YH, Carducci MA, et al. Chemohormonal Therapy in Metastatic HormoneSensitive Prostate Cancer: Long-Term Survival Analysis of the Randomized Phase III E3805 CHAARTED Trial. J Clin Oncol 2018;36:1080-7.
34. James ND, Sydes MR, Clarke NW, et al. Addition of docetaxel, zoledronic acid, or both to first-line longterm hormone therapy in prostate cancer (STAMPEDE): survival results from an adaptive, multiarm, multistage, platform randomised controlled trial. Lancet 2016;387:1163-77.

35. Fizazi K, Tran N, Fein L, et al. Abiraterone acetate plus prednisone in patients with newly diagnosed highrisk metastatic castration-sensitive prostate cancer (LATITUDE): final overall survival analysis of a randomised, double-blind, phase 3 trial. Lancet Oncol 2019;20:686-700.

36. James ND, de Bono JS, Spears MR, et al. Abiraterone for Prostate Cancer Not Previously Treated with Hormone Therapy. N Engl J Med 2017;377:338-51.

37. Rydzewska LHM, Burdett S, Vale CL, et al. Adding abiraterone to androgen deprivation therapy in men with metastatic hormone-sensitive prostate cancer: A systematic review and meta-analysis. Eur J Cancer 2017;84:88-101.

38. Davis ID, Martin AJ, Stockler MR, et al. Enzalutamide with Standard First-Line Therapy in Metastatic Prostate Cancer. N Engl J Med 2019;381:121-31.

39. Armstrong AJ, Szmulewitz RZ, Petrylak DP, et al. ARCHES: A Randomized, Phase III Study of Androgen Deprivation Therapy With Enzalutamide or Placebo in Men With Metastatic Hormone-Sensitive Prostate Cancer. J Clin Oncol 2019;37:2974-86.

40. Chi KN, Agarwal N, Bjartell A, et al. Apalutamide for Metastatic, Castration-Sensitive Prostate Cancer. N Engl J Med 2019;381:13-24.

41. Sathianathen NJ, Koschel S, Thangasamy IA, et al. Indirect Comparisons of Efficacy between Combination Approaches in Metastatic Hormone-sensitive Prostate Cancer: A Systematic Review and Network Meta-analysis. Eur Urol 2020;77:365-72.

42. Anido-Herranz U, Fernández-Núñez N, Afonso-Afonso J, et al. Chemotherapy management for unfit patients with metastatic castration-resistant prostate cancer. Clin Transl Oncol 2019;21:249-58.

43. Khalaf DJ, Annala M, Taavitsainen S, et al. Optimal sequencing of enzalutamide and abiraterone acetate plus prednisone in metastatic castration-resistant prostate cancer: a multicentre, randomised, open-label, phase 2, crossover trial. Lancet Oncol 2019;20:1730-9.

44. de Wit R, de Bono J, Sternberg CN, et al. Cabazitaxel versus abiraterone or enzalutamide in metastatic prostate cancer. N Engl J Med 2019;381:2506-18.

45. Smith MR, Saad F, Hussain M, et al. ARASENS: A phase 
3 trial of darolutamide in combination with docetaxel

for men with metastatic hormone-sensitive prostate cancer (mHSPC). J Clin Oncol 2018;36. doi: 10.1200/ JCO.2018.36.6_suppl.TPS383

46. Hofman MS, Lawrentschuk N, Francis RJ, et al. Prostatespecific membrane antigen PET-CT in patients with high-risk prostate cancer before curative-intent surgery or radiotherapy (proPSMA): a prospective, randomised, multicentre study. Lancet 2020;395:1208-16.

47. Vapiwala N, Hofman MS, Murphy DG, et al. Strategies for Evaluation of Novel Imaging in Prostate Cancer: Putting the Horse Back Before the Cart. J Clin Oncol 2019;37:765-9.

48. Murphy DG, Hofman MS, Azad A, et al. Going nuclear: it is time to embed the nuclear medicine physician in the prostate cancer multidisciplinary team. BJU Int 2019. [Epub ahead of print]. doi: 10.1111/bju.14814.

49. Thangasamy IA, Kwan EM, Teh J, et al. Novel agents for metastatic hormone-sensitive prostate cancer - a practice guide for urologists. BJU Int 2020;125:342-5.

50. Gillessen S, Attard G, Beer TM, et al. Management of Patients with Advanced Prostate Cancer: Report of the Advanced Prostate Cancer Consensus Conference 2019. Eur Urol 2020;77:508-47.

51. dela Rama F, Pratz C. Navigating Treatment of Metastatic Castration- Resistant Prostate Cancer: Nursing Perspectives. Clin J Oncol Nurs 2015;19:723-32.

52.Prettyman J. Personalizing Treatment In the Delivery of Care by Nurses To Patients with Prostate Cancer. Urol Nurs 2019;39:83-99.

53. Chambers SK, Frydenberg M, Dunn J. Quality of life considerations in the treatment of metastatic hormonesensitive prostate cancer. Lancet Oncol 2019;20:1469-71.

54. Gordon LG, Walker SM, Mervin MC, et al. Financial toxicity: a potential side effect of prostate cancer treatment among Australian men. Eur J Cancer Care (Engl) 2017;26:e12392.

55. Sathianathen NJ, Alarid-Escudero F, Kuntz KM, et al.

Cite this article as: Ong S, O'Brien J, Medhurst E, Lawrentschuk N, Murphy D, Azad A. Current treatment options for newly diagnosed metastatic hormone-sensitive prostate cancer-a narrative review. Transl Androl Urol 2021;10(10):3918-3930. doi: 10.21037/tau-20-1118
A Cost-effectiveness Analysis of Systemic Therapy for Metastatic Hormone-sensitive Prostate Cancer. Eur Urol Oncol 2019;2:649-55.

56. ClinicalTrials.gov. A Phase III Study for Patients With Metastatic Hormone-naïve Prostate Cancer (PEACE1) 2013. Available online: https://clinicaltrials.gov/ct2/show/ NCT01957436

57. Boevé LMS, Hulshof M, Vis AN, et al. Effect on Survival of Androgen Deprivation Therapy Alone Compared to Androgen Deprivation Therapy Combined with Concurrent Radiation Therapy to the Prostate in Patients with Primary Bone Metastatic Prostate Cancer in a Prospective Randomised Clinical Trial: Data from the HORRAD Trial. Eur Urol 2019;75:410-8.

58. Parker CC, James ND, Brawley CD, et al. Radiotherapy to the primary tumour for newly diagnosed, metastatic prostate cancer (STAMPEDE): a randomised controlled phase 3 trial. Lancet 2018;392:2353-66.

59. Emmett L, Willowson K, Violet J, et al. Lutetium (177) PSMA radionuclide therapy for men with prostate cancer: a review of the current literature and discussion of practical aspects of therapy. J Med Radiat Sci 2017;64:52-60.

60. Hofman MS, Violet J, Hicks RJ, et al. [(177)Lu]PSMA-617 radionuclide treatment in patients with metastatic castration-resistant prostate cancer (LuPSMA trial): a single-centre, single-arm, phase 2 study. Lancet Oncol 2018;19:825-33.

61. Hofman MS, Emmett L, Sandhu SK, et al. TheraP: A randomised phase II trial of 177Lu-PSMA-617 (LuPSMA) theranostic versus cabazitaxel in metastatic castration resistant prostate cancer (mCRPC) progressing after docetaxel: Initial results (ANZUP protocol 1603). J Clin Oncol 2020;38:5500.

62. ClinicalTrials.gov. In Men With Metastatic Prostate Cancer, What is the Safety and Benefit of Lutetium-177 PSMA Radionuclide Treatment in Addition to Chemotherapy (UpFrontPSMA). 2020. Available online: https://www.clinicaltrials.gov/ct2/show/NCT04343885 